\title{
ASIGNACIÓN DE FRECUENCIAS APROPIADAS A TRAVÉS DE UN MODELO MULTIOBJETIVO PARA UN SISTEMA BRT
}

\author{
Diego ARMANDo GalindRES GuANCHA ${ }^{1}$ \\ Jose AdAlberto Soto MEjía ${ }^{2}$ \\ SANDRA ESTRADA MEJÍA ${ }^{3}$
}

\section{RESUMEN}

En este artículo se aborda el problema de la búsqueda de frecuencias apropiadas de despacho de buses en un Sistema Integrado de Transporte Público (SITP). Se propone un modelo de optimización multiobjetivo para encontrar estas frecuencias de despacho de buses que transitan por un carril exclusivo en diferentes rutas. El modelo se aplicó a una reducción del SITP en la ciudad de Pereira (Risaralda, Colombia), el cual es del tipo Bus Rapid Transit (BRT), no obstante puede ser empleado en otro sistema BRT de mayor tamaño.

Las frecuencias se determinan de acuerdo a la demanda de viajes estimada en una estación de buses dada, la capacidad de los buses, y el tamaño de la flota disponible. Se resuelve una función multiobjetivo que minimiza el tiempo de parada en las estaciones, los costos económicos de las empresas operadoras, y considera una penalización cuando un usuario debe esperar por un segundo bus, por falta de cupo. Finalmente, se propone una metodología para escoger los ponderadores de la función objetivo.

El modelo fue resuelto con un Algoritmo Genético (AG). Las frecuencias de despacho fueron acotadas, para lograr una disminución del espacio de soluciones, obteniéndose de la solución intervalos de despacho operables. El modelo fue probado en diferentes escenarios con demanda baja, media y alta, lo que permitió evidenciar el impacto de estos en los tiempos de espera, tiempo de viaje promedio, y en general evidenciar los cambios en la calidad del servicio.

PALABRAS CLAVE: Bus Rapid Transit (BRT); frecuencias de despacho; modelo de optimización; Sistema Integrado de Transporte Público (SITP); tiempo de espera; tiempo de viaje.

\section{APPROPRIATE FREQUENCY ALLOCATION FOR A BRT SYSTEM THROUGH A MULTIOBJECTIVE MODEL}

\section{ABSTRACT}

This article addresses the search for adequate frequencies of bus dispatching in an Integrated Public Transportation Systems (IPTS). An optimization model is proposed to find these frequencies for bus dispatching travelling in different

1 Ingeniero Industrial, Estudiante de Maestría en Investigación Operativa y Estadística. Universidad Tecnológica de Pereira.

2 Ph.D. en Ingeniería Eléctrica, área Ingeniería de Computación. Universidad Tecnológica de Pereira.

3 Ph.D. en Ciencias Pedagógicas. Universidad Tecnológica de Pereira.

Autor de correspondencia: Galindres Guancha, D.A. (Diego Armando): Carrera 27 \#10-02 Barrio Alamos. Universidad Tecnológica de Pereira. Blq 5. piso 4, oficina de Proyectos especiales y Maestrias. / Tel.: 3218567297. Correo electrónico: diegogal@utp.edu.co
Historia del artículo:

Artículo recibido: 18-I-2016/ Aprobado: 29-XI-2016

Disponible online: 30 de febrero de 2017

Discusión abierta hasta abril de 2018 
exclusive lane routes. The model was applied to a reduction of the Integrated Public Transportation Systems (IPTS) in the city of Pereira (Risaralda, Colombia), which is of the Bus Rapid Transit (BRT) type. Nevertheless, the model can be applied to a larger BRT system.

The frequencies are determined according to an estimated travel demand in a given bus station, the capacity of the buses, and the size of the available fleet. There is resolution of a multi-objective function that minimizes downtimes at the stations, the economic costs of the business operators and takes into account a penalty that occurs when users must wait for a second bus. Finally, a methodology to choose the weights of the multi-objective function is shown.

The model was solved using a genetic algorithm (GA). Dispatch frequencies are bounded, achieving a reduction of the solution space and obtaining operable intervals.

KEYWORDS: Bus Rapid Transit (BRT); Dispatch frequency; Integrated Public Transportation Systems (IPTS); Optimization Model; Waiting time; Travel time.

\section{ATRIBUIÇÃO DE FREQUÊNCIAS APROPRIADAS ATRAVÉS DE UM MODELO MULTIOBJETIVO PARA UM SISTEMA BRT}

\section{RESUMO}

Neste artigo aborda-se o problema da procura de frequências apropriadas de envio de ônibus num Sistema Integrado de Transporte Público (SITP). Propõe-se um modelo de optimização multi - objetivo para encontrar estas frequências de envio de ônibus que transitam por um carril exclusivo em diferentes rotas. O modelo aplicou-se a uma redução do SITP na cidade de Pereira (Risaralda, Colômbia), o qual é do tipo ônibusRapid Transit (BRT), não obstante pode ser empregue em outro sistema BRT de maior tamanho.

As frequências determinam-se de acordo à demanda de viagens estimada numa estação de ônibus devido, a capacidade dos ônibus, e o tamanho da empresa disponível. Resolve-se uma função multi- objetivo que minimiza o tempo de parada nas estações, os custos económicos das empresas operadoras e considera uma penalização quando um usuário deve esperar por um segundo ônibus, por falta de vaga. Finalmente, propõe-se uma metodologia para escolher os ponderadores da função objetivo.

0 modelo foi resolvido com um Algoritmo Genético (AG). As frequências de envio foram disminuidas, para conseguir uma diminuição do espaço de soluções, obtendo da solução intervalos de envio operáveis. 0 modelo foi provado em diferentes cenários com demanda baixa, média e alta, o que permitiu evidenciar o impacto destes nos tempos de espera, tempo de viagem média, e em general evidenciar as mudanças na qualidade do serviço.

PALAVRAS-CHAVE: ÔnibusRapid Transit (BRT); Frequências de envio; Modelo de optimização; Sistema Integrado de Transporte Público (SITP); Tempo de espera; Tempo de viagem.

\section{INTRODUCCIÓN}

La planificación operativa de un Sistema Integrado de Transporte Público (SITP) es muy importante para una ciudad, ya que una mejora en este, repercute en la disminución de los tiempos de viaje esperados, impacta en la calidad y el costo del servicio, además, aumenta la confianza en sus usuarios y disminuye la posibilidad de preferir otro tipo de transporte. Es por eso que el desarrollo del transporte público es una de las prioridades de la administración pública, y también una de las vías más eficaces para resolver problemas de movilidad. (Luhua, Yin, y Xinkai, 2011).

El hecho de que los pasajeros, cotidianamente vivan situaciones de estrés, debido a que expe- 
rimentan condiciones no muy favorables al viajar, como la espera excesiva y la saturación del sistema por la demanda acumulada en las estaciones, hace pensar en mecanismos de planificación que permitan mejorar estas condiciones, teniendo en cuenta que el costo operativo sea razonable y que nuevas exigencias de los usuarios sean tenidas en cuenta (servicio rápido y confiable, amplia cobertura, servicio más barato y conductores seguros amistosos), (Rohani, Wijeyesekera, y Karim, 2013).

De acuerdo a lo anterior, Martínez, Mauttone, y Urquhart (2012) establecen que una definición apropiada de frecuencias mejoran las condiciones de los usuarios (tiempo de espera, tiempo de viaje) y las de los operadores (costo operacional determinado principalmente por el tamaño de la flota requerido).

En el presente trabajo de investigación se determinan las frecuencias de salida de la flota de buses para las rutas de una reducción del SITP Megabus, en la ciudad de Pereira. Este sistema es del tipo Bus Rapid Transit (BRT) (Cervero, 2013) y se considera la secuencia de las estaciones del sistema, los tiempos de recorrido entre ellas, la demanda de viajes en cada estación, dada por una matriz Origen-Destino y el tamaño de la flota. La metodología empleada puede extenderse a otro sistema BRT de mayor tamaño.

El modelo busca mejorar la calidad del servicio, disminuyendo el tiempo promedio de viaje de los pasajeros, el tiempo promedio de espera en una estación, los costos económicos de las empresas operadoras y considera una penalización cuando un usuario debe esperar por un segundo bus, por falta de cupo. Finalmente, se propone una metodología para escoger los ponderadores de la función multiobjetivo.

El artículo está organizado de la siguiente forma: primero se hace una revisión del estado del arte, luego se formula el modelo matemático, después se describe el algoritmo de resolución, luego se analizan los resultados y finalmente se dan las conclusiones y se proponen trabajos futuros.

\section{MARCO TEÓRICO}

La definición de frecuencias en un SITP es un problema que ha sido ampliamente abordado por diferentes investigadores. En Ceder (2015) se establece que el proceso de diseño y planificación de un SITP se debe considerar en cinco etapas diferentes, siendo la etapa de la definición de frecuencias apropiadas la segunda, después de haber definido los recorridos o rutas apropiadas, ya que estas dependen de la variación de la demanda. (Martínez et al., 2012). El problema de optimización de recorridos y frecuencias en sistemas de transporte público conocido como TNDP (Transit Network Design Problem), da solución a las dos etapas de planificación anteriormente mencionadas. En este problema, las rutas deben ser definidas de acuerdo a la infraestructura de calles y paradas, satisfaciendo una demanda determinada.

El problema de configuración de la frecuencia de una red de transporte TNFSP (Transit network frequency setting problem) es el que se trata en la presente investigación, donde el número total de buses necesarios para la operación de un SITP, depende principalmente de los intervalos de los tiempos de salida (inverso de las frecuencias). Los operadores de transporte público procuran proporcionar intervalos de salida máximos con el fin de reducir gastos de operación. Los pasajeros, por el contrario, requieren intervalos de salida mínimos con el fin de reducir el tiempo de espera y también el tiempo de viaje.

Cepeda, Cominetti, y Florian (2006) y Kov, Fukuday Yai (2011) abordan la anterior problemática modelando diferentes contextos. Por ejemplo, tienen en cuenta la congestión de tráfico en las calles y buscan mejorar el desempeño de cada ruta, expuesta a la variabilidad de los tiempos de recorrido debido a la congestión del tráfico, lo que afecta también al tiempo de espera de los usuarios y por tanto, su comportamiento dentro del sistema. En el contexto de la presente investigación, la congestión de las rutas no se tiene en cuenta ya que el sistema cuenta con un corredor propio haciendo que los tiempos de 
espera en las estaciones, debido al tránsito del bus, se puedan asumir como constantes.

Por otra parte, Qian, Luo, y Zeng (2005) aplican modelos reducidos en su complejidad matemática y consideran una carga máxima permitida en los buses, la cual condiciona las frecuencias de despacho. Se trata de reducir en la función objetivo solo la saturación de los pasajeros pero no tiene en cuenta los tiempos de paradas en estaciones, ni el tamaño de flota, condicionando solo las frecuencias máximas y mínimas permitidas.

En Luhua et al. (2011) se propone un modelo matemático simplificado, donde se maximiza el beneficio social económico neto. Se tienen en cuenta rutas compartidas para el cálculo del tiempo de espera. Se utiliza un factor para precisar la cantidad de personas que se bajan en una estación determinada, y a través de un tiempo promedio por persona se determina cuánto tiempo permanece el bus en una estación. Se introduce una razón que determina el porcentaje de pasajeros que deben hacer transferencia en un origen-destino determinado. Además se definen tiempos máximos de parada en las estaciones y factores de carga máximos en las rutas. La principal dificultad de este enfoque está relacionada con la parametrización de la información utilizada como insumo para el modelo.

Huang, Ren, y Liu (2013) consideran la demanda y su incertidumbre como un factor adicional que influye en la variación de los tiempos de recorridos, afectando directamente al tiempo que un bus se demora en una estación, por lo que incluyen en la función objetivo la disminución de la variabilidad de los tiempos de viaje esperados, con el fin de mejorar la confiabilidad del sistema. No obstante, estos esfuerzos por reducir la variabilidad de tiempo, están sujetos al nivel de caracterización que se tenga de la demanda potencial de viajes, de forma muy detallada y actualizada, lo que implica tener un sistema de información que se actualice constantemente con la información de la operación. En Ibeas, Alonso, dell'Olio, y Moura (2014) se tiene en cuenta la congestión en el sistema de transporte pú- blico y se considera demanda elástica. Proponen un modelo de optimización de frecuencias con flota de diferentes capacidades (diferente tamaño de buses), el objetivo es minimizar los costos operativos y de los usuarios, permitiendo analizar el impacto de la configuración de despacho sobre los cambios en el modo de transporte de los usuarios.

En Y. Li y Si (2014), se presenta un modelo de optimización multiobjetivo que incluye el costo de viaje y el beneficio de la compañía de tránsito, donde se limita el tiempo de espera aceptable a un valor fijo; sobre este valor se genera insatisfacción, además, contempla la insatisfacción de los pasajeros que van de pie. Los costos de tiempo son convertidos a unidades monetarias, lo cual implica estimar el parámetro asociado con el costo de una unidad de tiempo.

En Herbon y Hadas (2015) se presenta un enfoque que combina tanto los costos de pasajeros como los de operador dentro de un modelo generalizado. El modelo busca encontrar la combinación de frecuencias que maximiza los beneficios, teniendo en cuenta costos de espera, de hacinamiento y los costos del operador relacionados con el tamaño del vehículo, los asientos vacíos y las ventas perdidas. Se asume una demanda estocástica, la saturación de pasajeros es modelada bajo el concepto de demanda agregada, es decir, que esta tiene en cuenta no solo la estación origen sino también las demás estaciones que son intermedio para alcanzar un destino. Sin embargo, en la formulación no se incluye un factor que penalice la demanda insatisfecha que no alcanza a subir en los buses y debe esperar por otro bus.

Las anteriores formulaciones llevan implícito un sub-modelo de asignación, con el fin de representar adecuadamente la forma en que los pasajeros utilizan la oferta disponible (en términos de infraestructura, frecuencias de línea y otras normas de funcionamiento predefinidos), para viajar a través de la red de tránsito (Cortés, Jara-Moroni, Moreno, y Pineda, 2013). Este tipo de modelos son complejos en su resolución porque presentan variables de decisión que no solo tienen que ver con la frecuencia sino también con el comportamiento de los usuarios 
dentro del sistema. S. Li, Ye, y Wang (2016) utilizan la ley de gravitación para analizar la relación entre el volumen de pasajeros y las diferentes frecuencias del servicio. El modelo es parametrizado por la información obtenida en una encuesta que indaga por los factores que más influencian la escogencia del modo (bus, metro) de transporte: costo de pasaje, tiempo de viaje, comodidad, conveniencia y distancia del hogar a la estación de buses o del metro.

Para la presente investigación, la asignación de pasajeros es proporcional al nivel de servicio (frecuencia) de cada ruta, asumiendo que estos (los pasajeros) carecen de una estrategia a priori para escoger el bus como las que se consideran en Spiess y Florian (1989), Leurent, Chandakas, y Poulhès (2012), Verbas, S. Mahmassani, y F. Hyland (2016). Esto se debe principalmente al tamaño del SITP considerado, dado que para el pasajero no representa una diferencia significativa, en tiempo de viaje, el escoger una u otra ruta factible hacia su destino. Al no tenerse en cuenta el efecto de la congestión en las rutas, el tiempo de parada en las estaciones es el factor que más impacta el tiempo de viaje, como lo describen Wirasinghe y Szplett (1984) y Lam, Cheung y Poon (1998). Todo lo anterior permite simplificar la formulación del modelo y por ende la complejidad de su solución.

Así, el modelo multiobjetivo propuesto minimiza los costos de operación y tiempos de viaje, penalizando en la función objetivo la cantidad de pasajeros que deben esperar por un segundo bus, enfocándose en equilibrar factores como tiempo de parada en las estaciones (en función de la cantidad de pasajeros que se suben y se bajan en cada estación) y el costo de la operación, considerando como restricciones el tamaño de flota disponible, dada una matriz origen-destino.

\section{MATERIALES Y MÉTODOS}

La estructura del SITP en estudio cuenta con 3 rutas (ver Figura 1). La ruta 1 comparte las mismas estaciones en ambos recorridos (ida y regreso); en la ruta 2 , ambos recorridos comparten algunas estaciones (inicio y final de cada recorrido). La ruta 3 es circular, pero se divide en la mitad (nodo 13), para definir un recorrido de ida y regreso.

\section{Figura 1. Red de rutas del sistema modelado}

Fuente: elaboración propia
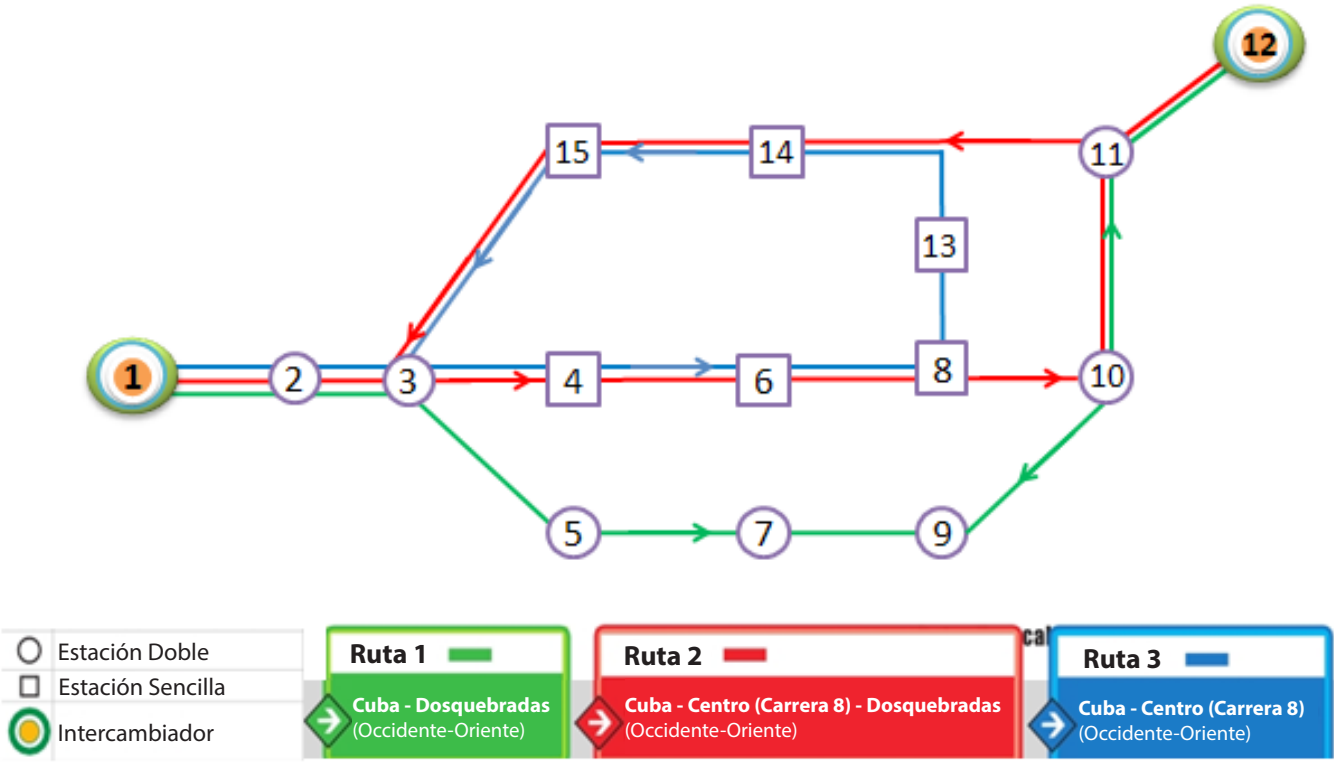


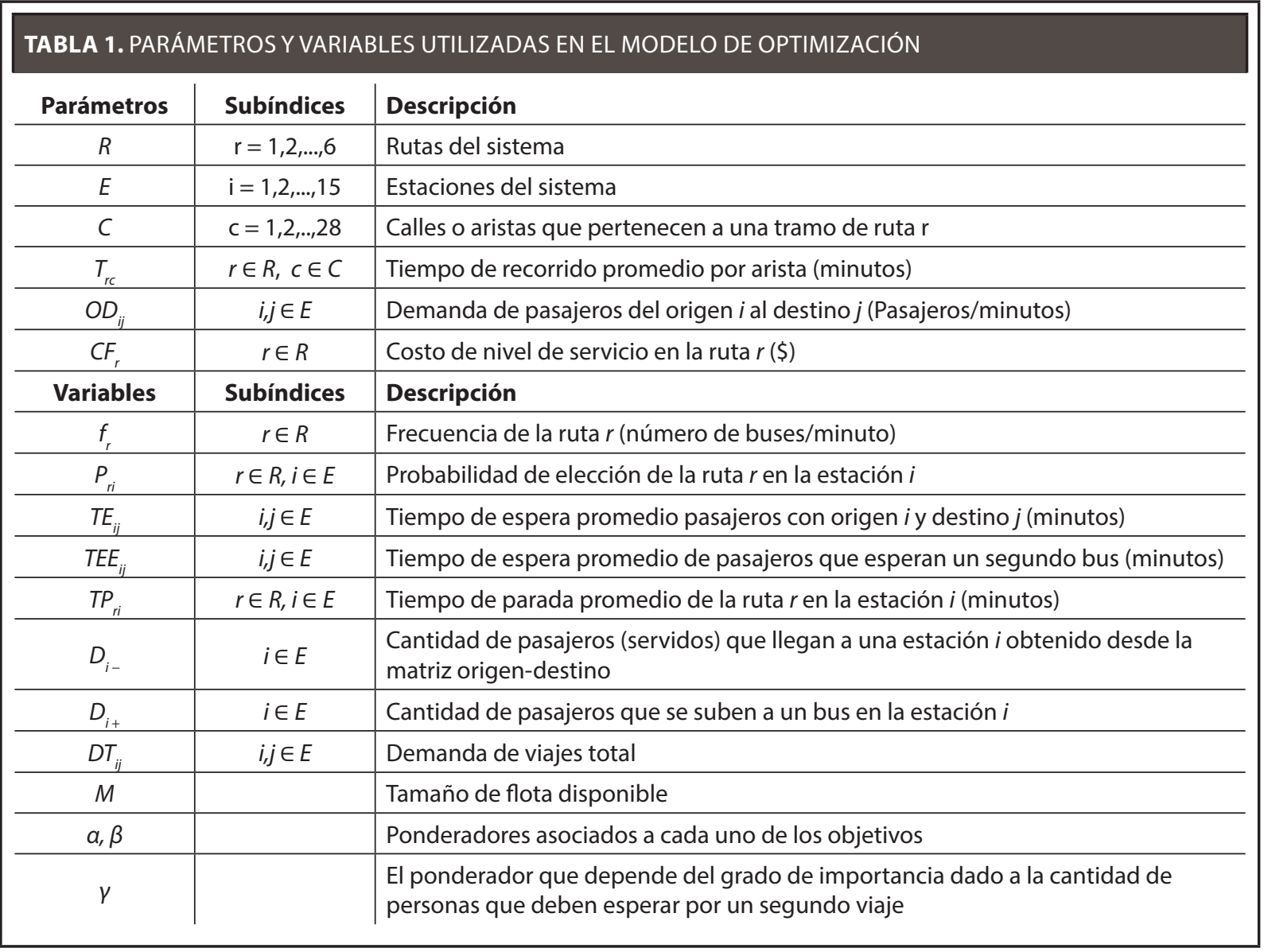

Siguiendo el modelo matemático propuesto por Luhua et al. (2011) e incluyendo la cantidad de pasajeros que deben esperar por un segundo bus se define una estructura topológica a través de los grafos $R=(E, C)$, los cuales representan cada uno las tres rutas del sistema considerado, divididas en dos sentidos, ida y regreso, para un total de seis grafos. Los grafos 1 (ruta 1 de ida) y 4 (ruta 1 de regreso); 2 (ruta 2 de ida) y 5 (ruta 2 de regreso); 3 (ruta 3 de ida) y 6 (ruta 3 de regreso) conforman cada uno un ciclo completo.

La oferta del SITP está representada por las frecuencias a encontrar que se les asignara a los grafos $R$. La demanda está dada por los usuarios (pasajeros) que necesitan realizar viajes entre diferentes estaciones del sistema. En la Tabla 1 se muestran los parámetros y variables utilizados.
La distribución de la demanda dentro del sistema no está condicionada por una estrategia de asignación, es decir, se asume que los pasajeros abordan el primer bus factible que les sirva para llegar a su destino. Por tanto, la demanda en una estación se distribuye en las rutas proporcionalmente al valor de la frecuencia que se le asigne, de acuerdo con la siguiente expresión:

$$
P_{r i}=\frac{f_{r}}{\sum_{r \in R} f_{r}} \quad r \in R, \forall i \in E
$$

La anterior expresión muestra que si una estación está compartida por más de una ruta $R$ del mismo sentido, entonces la demanda será distribuida proporcionalmente al nivel de servicio que ofrezcan, $0<P_{r i}<1$, y en otro caso $P_{r i}=1$, cuando hay una sola ruta para escoger. Por tanto, se asume que el usuario no considera información sobre los tiempos 
de viaje a bordo, las frecuencias de cada ruta, y los tiempos de espera, para hacer una elección a priori de la ruta que desea utilizar.

En el presente caso, la demanda en cada estación se toma como conocida. Lo anterior implica que el modelo calcula las frecuencias apropiadas para un instante de tiempo, sin tener en cuenta la variabilidad de la demanda en el tiempo. Por tanto se consideran diferentes escenarios (demanda baja, media y alta) y se calculan frecuencias apropiadas para cada uno.

Al tenerse en cuenta un modelo de asignación simplificado de usuarios a rutas, el problema de calcular frecuencias apropiadas para todo el sistema se reduce a minimizar el tiempo de espera en cada estación.

El tiempo promedio de espera $\left(T E_{i j}\right)$, en cada estación se obtiene de la distribución de tiempos entre llegadas de los buses, dado por la siguiente expresión:

$$
T E_{i j}=\frac{1}{2 \sum_{r \in R} f_{r}} \quad r \in R, \forall i, j \in E
$$

La expresión anterior asume que una vez encontradas las frecuencias apropiadas los tiempos entre llegadas de los buses permanecen constantes, como lo propuesto por Spiess y Florian (1989). Donde el tiempo de espera promedio de un pasajero, se calcula teniendo en cuenta las diferentes las rutas factibles que el usuario pueda abordar.

Para cualquier pasajero el tiempo de espera estará entre $\left[0 y \frac{1}{\sum_{r \in R} f_{r}} ; r \in R, \forall i, j \in E\right]$.

Nótese que este tiempo depende estrictamente del nivel de servicio ofrecido $\left(f_{r}\right)$. Así el tiempo de espera por un segundo bus $\left(T E E_{i j}\right)$ resulta de sumar el tiempo de espera promedio $\left(T E_{i j}\right)$ más el tiempo máximo de espera, $\frac{1}{\sum_{r \in R} f_{r}}$.

El tiempo invertido por los pasajeros al abordar el bus, dirigirse a su destino (parando en todas las estaciones intermedias) y bajarse en la estación destino, se llamará tiempo de viaje. El tiempo de recorrido entre estaciones se denotará como $T_{r c}$. Los tiempos de parada en las estaciones $\left(T P_{r i}\right)$, varían de acuerdo a las frecuencias asignadas.

La frecuencia se define como un conjunto de valores $f_{r}=\left\{\varphi_{1}, \varphi_{2}, \ldots \varphi_{m}\right\}$, donde cada elemento $\varphi_{i^{\prime}}$ es no negativo y representa una posible frecuencia a ser asignada a una ruta $R$ compuesta por los grafos 1 y 4,2 y 5,3 y 6 .

\subsection{Modelo de optimización}

La formulación siguiente minimiza una combinación de los objetivos correspondientes a usuarios y operadores, ponderados por coeficientes de importancia que además deben realizar la conversión a unidades comunes.

El objetivo de los usuarios se representa como la minimización de los tiempos de viaje promedio, (Espera en una estación $T E_{i j}$, Tiempo de espera por un segundo bus $T E E_{i j}$, Tiempo de recorrido entre estaciones $T_{r c}$, Tiempo de parada en estaciones $T P_{r i}$ ) para todo par de estaciones factibles en las rutas $R$. Los ponderadores $\alpha, \beta$, asociados a cada uno de los objetivos dependen de la prioridad que se le quiera dar a los intereses del usuario y del operador, respectivamente. El ponderador $\gamma$ va a depender del grado de importancia con que se quiera reducir la cantidad de personas que deben esperar por un segundo viaje. El objetivo de los operadores está representado por el costo de las frecuencias (o nivel de servicio que estas representen) $C F_{r^{*}}$

$\alpha, \beta$ afectan el modelo en cualquier escenario de demanda, mientras que $\gamma$ solo es útil a partir de un nivel de demanda determinado. La formulación es la siguiente:

$$
\begin{array}{r}
\min \alpha\left(\sum_{i, j \in E} T E_{i j}+\gamma \sum_{i, j \in E} T E E_{i j}+\sum_{r \in R, c \in C} T_{r c}+\right. \\
\left.\sum_{r \in R, i \in E} T P_{r i}\right)+\beta \sum_{r \in R} C F_{r} f_{r}
\end{array}
$$

Sujeto a: 


$$
\begin{gathered}
\sum_{i \in E} D_{i-}-\sum_{i \in E} D_{i+}=O D_{i j} \quad \forall i, j \in E \\
D T_{i j} \leq f_{r} T E_{i j} \quad \forall i, j \in E, \quad r \in R \\
D T_{i j}>0 \quad \forall i, j \in E \\
\sum_{r \in R}\left(\sum_{c \in C} T_{r c}+\sum_{i \in E} T P_{r i}\right) f_{r} \leq M \quad \forall C, E \in R \\
\left(\sum_{c \in C} T_{r c}+\sum_{i \in E} T P_{r i}\right) f_{r} \geq 1 \quad \forall C, E \in R
\end{gathered}
$$

En la Ecuación (4), $D_{i-}$ es la cantidad de pasajeros (servidos) que llegan a una estación $i$, lo cual está dado por la matriz origen-destino. $D_{i+}$, es la cantidad de pasajeros que se suben a un bus y se calcula como, $D_{i+}=P_{r i} O D_{i j}$. En la Ecuación (5) se ofrece un nivel de servicio acorde con la cantidad de demanda en el sistema. En la Ecuación (7) se restringe el nivel de servicio de acuerdo al tamaño de flota disponible $(M)$. En la Ecuación (8) se asegura que al menos haya un bus en cada ruta.

\subsection{Aplicación del algoritmo genético}

La solución al sistema de Ecuaciones $\mathbf{3}$ a 8 define una frecuencia para cada uno de los seis grafos de recorridos 1 y 4, 2 y 5,3 y 6 . Al discretizar los valores factibles de las frecuencias, se obtiene un problema de optimización combinatorial cuyo espacio de búsqueda tiene un tamaño exponencial $\left|f_{c}\right|^{r}$ (Martínez et al., 2012).

Dada la complejidad no lineal del problema y su estructura multiobjetivo, enfoques algorítmicos, como los basados en descomposición del problema o los métodos de gradiente, suelen no ser los más apropiados, (Huang et al., 2013). En el presente trabajo se buscaron soluciones a través de una metaheurística basada en Algoritmos Genéticos (AG) (Holland, 1992) la cual permite encontrar soluciones para problemas no lineales utilizando la librería Pyevolve de Python (Perone, C. S., 2009). A continuación se presentan algunas generalidades del AG utilizado.

Codificación. Cada ruta $R$ debe tener asignado a una frecuencia $f_{r^{\prime}}$. Así un individuo (un cromosoma) de la población estará compuesto por tres valores de frecuencia posibles (el valor de la frecuencia de ida es igual a la de regreso para cada una de las rutas).

Población inicial. Por defecto, se crea una lista de 500 cromosomas cuyo tamaño coincide con la cantidad de rutas, es decir 3 . El valor asignado a cada gen oscila entre 3,5 a 15,5 minutos (convertidos a frecuencias), limites que tiene actualmente la operación real. Los valores a asignar tienen un intervalo de discretización de 0,5 minutos, por tanto, se obtienen 25 posibles valores que podría tomar $f_{r}$ en cada gen. Consistente con lo anterior se inicia cada cromosoma con valores aleatorios uniformemente distribuidos para lo cual se utilizó el generador de números aleatorios IntegerRangeMutator de Pyevolve.

Función de adaptación. Se calcula la función objetivo dada por la Ecuación (3) de acuerdo con las frecuencias asignadas, y se penaliza la función objetivo de los individuos de la población que excedan alguna de las restricciones. Ecuaciones 5, 7 y 8.

Selección. Hace que las soluciones con mejor función objetivo tengan más posibilidades de sobrevivir y reproducirse. Por defecto, se eligieron los mejores 20 individuos por el método GRankSelector de Pyevolve.

Cruzamiento. La producción de nuevas soluciones se hizo combinando las mejores soluciones existentes con cruzamiento de punto fijo.

Criterio de convergencia. Se hizo uso del método evolve(frec_stats=5) de Pyevolve el cual analiza las estadísticas de las últimas 5 generaciones, y si no cambian por 20 generaciones se detiene el proceso. El tiempo de cómputo promedio fue de 30,405 segundos, y la incumbente final encontrada se obtuvo después de 2016 iteraciones.

\section{EXPERIMENTACIÓN, RESULTADOS Y DISCUSIÓN}

Se corrieron 3 escenarios bajo una demanda alta, media y baja. Se compararon las frecuencias encontradas, en cada escenario, con las que actualmente opera el sistema. A partir de lo anterior se pudo 
comparar las configuraciones de operación (combinaciones de frecuencias), en términos del tamaño de flota, tiempos de viaje promedio (se toma como referencia el tiempo de un viaje para recorrer un ciclo completo en cada ruta) y los pasajeros esperando el segundo bus. El sistema base para la modelación fue mostrado en la sección III, Figura 1.

Los intervalos de tiempo entre salidas, para cada uno de los escenarios, se obtuvieron calculando el inverso de las frecuencias encontradas, al resolver el modelo dado en las Ecuaciones $\mathbf{3}$ a 8 .

Los resultados se muestran en la Tabla 2. Con fines de comparación se muestran también los intervalos de salidas que se aplican actualmente en el sistema Megabus.

TABLA 2. INTERVALOS DE SALIDAS A PARTIR DE LAS FRECUENCIAS ENCONTRADAS COMPARADOS CON LOS ACTUALES

\begin{tabular}{c|c|c|c|c} 
Demanda & $\begin{array}{c}\text { Intervalos entre } \\
\text { salidas (min) }\end{array}$ & R1 & R2 & R3 \\
\hline \multirow{2}{*}{ Alta } & AG & 4,5 & 6,0 & 5,0 \\
\hline \multirow{2}{*}{ Media } & Actual & 4 & 4 & 6 \\
\hline \multirow{2}{*}{ Baja } & AG & 5,5 & 9,5 & 4,0 \\
\cline { 2 - 5 } & Actual & 6 & 6 & 5 \\
\cline { 2 - 5 } & AG & 11 & 15,5 & 15,5 \\
\hline
\end{tabular}

La Tabla 3, muestra cómo todas las soluciones obtenidas a través del modelo de optimización (resuelto mediante AG) tienen una función objetivo menor (con respecto al tiempo promedio de viaje y el tamaño de flota requerido) comparado con la estrategia actual de operación.

En el escenario de demanda alta (ver Tabla 3), actualmente se utilizarían en el sistema reducido, usando las actuales reales frecuencias de despacho, más buses (32), lo cual repercute en menores tiempos de viaje, si se comparan ambas configuraciones. Sin embargo, como se observa la Tabla 4, se encuentra que la diferencia entre el número de personas que deben esperar un segundo bus, bajo ambos tamaños de flota es solo de 3 pasajeros, en el total de las tres rutas. Lo anterior está indicando que la actual estrategia está sobre dimensionando el número de buses bajo el escenario de demanda alta.

En el escenario de demanda media ocurre lo contrario. La configuración con las frecuencias encontradas con el AG (ver última columna de la Tabla 3) utiliza 3 buses por encima de la configuración actual, lo cual disminuye los tiempos de viaje promedio en las 3 rutas. No obstante lo anterior, en este caso sí existe una diferencia significativa entre el escenario de demanda media y frecuencia AG y el actual, disminuyendo el número de pasajeros que tienen que esperar un bus de 95 a 53 (ver Tabla 4, última columna).

\begin{tabular}{|c|c|c|c|c|c|c|}
\hline \multirow[t]{2}{*}{ Demanda } & \multirow[t]{2}{*}{ Configuración } & \multirow[t]{2}{*}{ F.O } & \multicolumn{3}{|c|}{$\begin{array}{l}\text { Tiempo promedio } \\
\text { Viaje (min) }\end{array}$} & \multirow{2}{*}{$\begin{array}{c}\text { Tamaño de Flota } \\
\text { Requerido }\end{array}$} \\
\hline & & & R1 & $\mathrm{R} 2$ & R3 & \\
\hline \multirow{2}{*}{ Alta } & AG & 727.376 & 57,5 & 51,6 & 43,4 & 28 \\
\hline & Actual & 757.082 & 56,8 & 50,7 & 42,6 & 32 \\
\hline \multirow{2}{*}{ Media } & AG & 506.655 & 53,5 & 43,8 & 40,0 & 22 \\
\hline & Actual & 589.230 & 54,5 & 44,6 & 41,5 & 19 \\
\hline \multirow{2}{*}{ Baja } & AG & 324.530 & 48,4 & 41,0 & 45,3 & 8 \\
\hline & Actual & 330.339 & 47,2 & 39,5 & 42,6 & 9 \\
\hline
\end{tabular}


TABLA 4. PASAJEROS ESPERANDO EL SEGUNDO

BUS PARTIR DE LAS FRECUENCIAS ACTUALES $Y$

LAS ENCONTRADAS CON EL AG, EN DIFERENTES ESCENARIOS

\begin{tabular}{c|c|c|c|c|c|}
\multirow{2}{*}{ Demanda } & \multirow{2}{*}{ Frecuencias } & \multicolumn{4}{|c}{ Pasajeros esperando el } \\
& & $\mathrm{R} 1$ & $\mathrm{R} 2$ & $\mathrm{R} 3$ & Total \\
\cline { 2 - 6 } Alta & AG & 273 & 43 & 228 & 544 \\
\cline { 2 - 6 } & Actual & 238 & 46 & 263 & 547 \\
\hline \multirow{3}{*}{ Media } & AG & 53 & 0 & 0 & 53 \\
\cline { 2 - 6 } & Actual & 61 & 0 & 34 & 95 \\
\hline \multirow{2}{*}{ Baja } & AG & 0 & 0 & 0 & 0 \\
\cline { 2 - 6 } & Actual & 0 & 0 & 0 & 0 \\
\hline
\end{tabular}

El escenario de menor demanda muestra poca diferencia entre las dos configuraciones (función objetivo, tiempo promedio de viaje, tamaño de flota y número de pasajeros esperando un segundo bus). Lo anterior se debe a que bajo este escenario no hay puntos críticos (saturación de demanda de pasajeros) que eleven los costos de los usuarios por tener que esperar un segundo bus o de los operadores por tener que ofrecer intervalos de salida más pequeños.

Se puede observar que al resolver el modelo mediante el AG, se obtiene un panorama estratégico de operación, que puede mejorar el uso de los recursos disponibles. No necesariamente con una demanda alta se debe utilizar la máxima flota disponible; existen otras configuraciones de despacho que con menos flota en uso, proporcionan un nivel de servicio similar, por ejemplo, al lograr el mismo promedio de personas que deben esperar por un segundo bus.

En la Tabla 5, para el escenario de demanda alta, se muestra el impacto de diferentes valores en los coeficientes $\alpha, \beta, \gamma$, de la función objetivo (ver Ecuación (3)) sobre el número de pasajeros esperando un segundo bus y el tamaño de la flota.

Si $\alpha=\beta=1$ los objetivos del usuario y el operador respectivamente, tienen la misma prioridad. El ponderador $\gamma$ determina el nivel de servicio en el escenario de demanda alta, aumentando el tamaño de flota y disminuyendo la cantidad de pasajeros promedio que deben esperar un segundo bus. De forma similar, al variar $\alpha$ y $\beta$ se producen contrastes en ambas variables analizadas. Note que en la Ecuación (3), $\alpha$, afecta a todos los escenarios, ya sean de demanda baja, media y alta; mientras que el parámetro $\gamma$, afecta solo al tiempo de espera de un segundo bus, $T E E_{i j}$ situación que se da en escenarios de demanda alta.

TABLA 5. VARIACIÓN DE LOS RESULTADOS DEL MODELO RESPECTO DE COEFICIENTES UTILIZADOS

\begin{tabular}{|c|c|c|c|c|c|}
\hline \multicolumn{3}{|c|}{ Ponderador } & Intervalos & \multirow{2}{*}{$\begin{array}{c}\text { Pasajeros } \\
\text { esperando el } \\
\text { segundo bus }\end{array}$} & \multirow{2}{*}{$\begin{array}{c}\text { Tamaño } \\
\text { Flota }\end{array}$} \\
\hline$\alpha$ & $\beta$ & $\gamma$ & $\mathrm{R} 1$ / R2 / R3 & & \\
\hline \multirow{2}{*}{1} & \multirow{2}{*}{1} & 3 & $4,5 / 6 / 5$ & 544 & 28 \\
\hline & & 5 & $3,5 / 5 / 4$ & 437 & 34 \\
\hline \multirow{2}{*}{2} & \multirow{2}{*}{1} & 3 & $3,5 / 5 / 3,5$ & 417 & 36 \\
\hline & & 5 & $3,5 / 5 / 3,5$ & 417 & 36 \\
\hline \multirow{2}{*}{1} & \multirow{2}{*}{2} & 3 & $4,5 / 5,5 / 7$ & 625 & 24 \\
\hline & & 5 & $4,5 / 6 / 5$ & 543 & 28 \\
\hline
\end{tabular}

En este artículo se propone como una posible estrategia metodológica para escoger de manera apropiada el valor de los ponderadores $\alpha, \beta, \gamma$, graficar el comportamiento de las variables analizadas (pasajeros esperando el segundo bus y Tamaño flota) como se muestra en la Figura 2.

\section{Figura 2. Comportamiento de las variables analizadas} para cada variación de $a \beta \gamma$

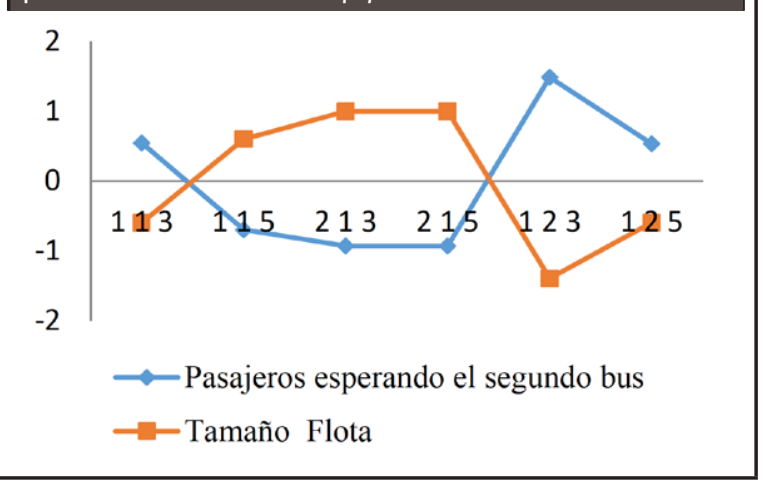


Para evitar el impacto de la magnitud de los valores y de las unidades de medición, los valores de "Pasajeros esperando el segundo bus" y "Tamaño Flota", dados en la Tabla 5 fueron estandarizados (se restó al valor original de la variable su valor promedio y se dividió por la desviación estándar).

Se calculó el promedio de 'Pasajeros esperando un segundo bus' y su desviación estándar; con ellos se estandarizó el valor para cada combinación de valores asignados a los ponderadores $\alpha, \beta, \gamma$. Así en la Figura 2, la abscisa 113, corresponde a $\alpha=1, \beta=1$, $\gamma=3$. En la ordenada se encuentra el valor de la variable estandarizada.

En la Figura 2, se puede ver la relación entre dos variables analizadas y su naturaleza de conflicto (al reducir el número de pasajeros esperando por el segundo bus, se aumenta el tamaño de la flota utilizado y viceversa). La gráfica muestra además cruces en los cuales se encuentra una combinación de ponderadores, $\alpha, \beta, \gamma$, que tienden a equilibrar los intereses del usuario y del operador. Así, bajo una demanda alta, los ponderadores de compromiso entre las partes, se obtienen promediando uno a uno, cada par de ponderadores entre los que se da un cruce, obteniéndose para el primer cruce $\alpha=1, \beta=1, \gamma=4$ o para el segundo cruce $\alpha=1.5, \beta=1.5, \gamma=4$. En ambos casos se obtiene 526 pasajeros esperando el segundo bus y un tamaño de flota requerido de 29 buses.

\section{CONCLUSIONES Y TRABAJO FUTURO}

Los intervalos de tiempo de salida, definidos a través de frecuencias apropiadas, son claves para el mejoramiento de la calidad del transporte público. La definición de frecuencias debe ser acorde a lo que esperan las dos partes implicadas, usuarios y operadores. Para los primeros se tuvo en cuenta la reducción del tiempo de viaje promedio y la cantidad de usuarios que deben esperar por un segundo bus. Para los operadores la reducción del costo de la operación medido por el tamaño de la flota.

Los resultados obtenidos a través del AG muestran que con la configuración AG (ver Tabla 3), no siempre se obtiene el menor tiempo de via- je promedio (ver escenario de demanda alta), dado que esta configuración utiliza menos buses que la configuración actual. Sin embargo, se observa que en el escenario de demanda media con tamaños de flota similares, 22 y 19 buses para la configuración AG y la actual, respectivamente (ver Tabla 3) sí se da una marcada reducción del número de pasajeros esperando un segundo bus, de 53 pasajeros encontrados según las frecuencias obtenidas por AG y los 95 pasajeros que esperan un segundo bus, con las frecuencias actuales (ver Tabla 4). El anterior resultado muestra que se ha dado una mejor distribución a la flota en todas las rutas, hasta hacerse cero el número de pasajeros esperando un segundo bus en las rutas R2 y R3, en el escenario de demanda media.

Como trabajo futuro se evaluará el modelo propuesto en el sistema ampliado de MEGABUS. Se deberán abordar investigaciones asociadas con la estimación de la demanda de los pasajeros y su comportamiento dentro del sistema, dada su importancia en la estimación de las frecuencias.

\section{AGRADECIMIENTOS}

A la Maestría de Investigación Operativa y Estadística de la Universidad Tecnológica de Pereira, por brindar todas las condiciones necesarias para desarrollar el trabajo en el mejor ambiente, a COLCIENCIAS por ser el ente financiador de la investigación y a MEGABUS S.A. por su colaboración y disposición al brindar información requerida.

\section{REFERENCIAS}

Ceder, A. (2015). Public Transit Planning and Operation: Modeling, Practice and Behavior, Second Edition. CRC Press.

Cepeda, M.; Cominetti, R.; Florian, M. (2006). A frequencybased assignment model for congested transit networks with strict capacity constraints: characterization and computation of equilibria. Transportation Research Part B: Methodological, 40(6), pp. 437-459. [Online] Disponible en: https://doi.org/10.1016/j. trb.2005.05.006 
Cervero, R. (2013). Bus rapid transit (BRT): An efficient and competitive mode of public transport. IURD Working Paper 2013-01. [Online] Disponible en: http://escholarship.org/uc/item/4sn2f5wc.pdf

Cortés, C. E.; Jara-Moroni, P.; Moreno, E.; Pineda, C. (2013). Stochastic transit equilibrium. Transportation Research Part B: Methodological, 51, pp. 29-44. [Online] Disponible en: https://doi.org/10.1016/j.trb.2013.02.001

Herbon, A.; Hadas, Y. (2015). Determining optimal frequency and vehicle capacity for public transit routes: A generalized newsvendor model. Transportation Research Part B: Methodological, 71, pp. 85-99. [Online] Disponible en: https://doi. org/10.1016/j.trb.2014.10.007

Holland, J. H. (1992). Adaptation in Natural and Artificial Systems: An Introductory Analysis with Applications to Biology, Control and Artificial Intelligence. Cambridge, MA, USA: MIT Press.

Huang, Z.; Ren, G.; Liu, H. (2013). Optimizing Bus Frequencies under Uncertain Demand: Case Study of the Transit Network in a Developing City, Mathematical Problems in Engineering, e375084. [Online] Disponible en: https://doi.org/10.1155/2013/375084, $10.1155 / 2013 / 375084$

Ibeas, A.; Alonso, B.; dell'Olio, L.; Moura, J. L. (2014). Bus Size and Headways Optimization Model Considering Elastic Demand. Journal of Transportation Engineering, 140(4), 04013021. [Online] Disponible en: https:// doi.org/10.1061/(ASCE)TE.1943-5436.0000641

Lam, W.H.K.; Cheung, C.Y.; Poon, Y.F. (1998), A study of train dwelling time at the hong kong mass transit railway system. J. Adv. Transp., 32, pp. 285-295. [Online] Disponible en: doi:10.1002/atr.5670320303

Leurent, F.; Chandakas, E.; Poulhès, A. (2012). A Passenger Traffic Assignment Model with Capacity Constraints for Transit Networks. Procedia - Social and Behavioral Sciences, 54, pp. 772-784. [Online] Disponible en: https://doi.org/10.1016/j.sbspro.2012.09.794

Li, S.; Ye, Z.; Wang, C. (2016). Optimization Model for TownVillage Bus Service Frequency Design. En CICTP2016, pp. 864-875. [Online] Disponible en: http://ascelibrary.org/doi/abs/10.1061/9780784479896.079

Li, Y.; Si, B. (2014). The Optimization Model of Urban Transit Departure Frequency. En: H. Xia y Y. Zhang (Eds.), The 2nd International Symposium on Rail Transit Comprehensive Development (ISRTCD) Proceedings (pp. 379-385). Berlin, Heidelberg: Springer Berlin
Heidelberg. [Online] Disponible en: https://doi. org/10.1007/978-3-642-37589-7_36

Luhua, S.; Yin, H.; Xinkai, J. (2011). Study on Method of Bus Service Frequency Optimal Model Based on Genetic Algorithm. Procedia Environmental Sciences, 10, Part A, 869-874. [Online] Disponible en: https:// doi.org/10.1016/j.proenv.2011.09.139

Martínez, H.M.; Mauttone, A.; Urquhart, M.E. (2012). Formulación y metaheurística para el problema de la determinación de frecuencias en el transporte colectivo público. [Online] Disponible en: http://www.din. uem.br/ ademir/sbpo/sbpo2012/pdf/arq0145.pdf

Perone, C.S. (2009). Pyevolve: a Python open-source framework for genetic algorithms. ACM SIGEVOlution, 4(1), pp. 12-20.

Qian, Y.; Luo, J.; Zeng, J. (2005). Bus Departure Interval Optimization Considering Crowing Cost.

Rohani, M.M.; Wijeyesekera, D.C.; Karim, A.T. A. (2013). Bus Operation, Quality Service and The Role of Bus Provider and Driver. Procedia Engineering, 53, pp. 167-178. [Online] Disponible en: https://doi. org/10.1016/j.proeng.2013.02.022

Spiess, H.; Florian, M. (1989). Optimal strategies: A new assignment model for transit networks. Transportation Research Part B: Methodological, 23(2), pp. 83-102. https://doi.org/10.1016/0191-2615(89)90034-9

Verbas, Ö.; Mahmassani, H.S.; Hyland, M.F. (2016). Gapbased transit assignment algorithm with vehicle capacity constraints: Simulation-based implementation and large-scale application. Transportation Research Part B: Methodological, 93, Part A, 1-16. [Online] Disponible en: https://doi.org/10.1016/j. trb.2016.07.002

Wirasinghe, S. C.; Szplett, D. (1984). An investigation of passenger interchange and train standing time at LRT stations: (ii) Estimation of standing time. J. Adv. Transp., 18, pp. 13-24. [Online] Disponible en: doi:10.1002/atr.5670180103

\section{PARA CITAR ESTE ARTÍCULO TO REFERENCE THIS ARTICLE / PARA CITAR ESTE ARTIGO /}

Galindres Guancha, D.A.; Soto Mejía, J.A.; Estrada Mejía, S. (2016). Asignación de frecuencias óptimas a través de un modelo multiobjetivo para un sistema BRT. Revista EIA, 13(26), julio-diciembre, pp. 141-152. [Online]. Disponible en: https://doi.org/10.24050/reia.v13i26.743 